\title{
Argyria From a Topical Home Remedy
}

\author{
Ashley M. Reader, DO; Derek Dillon, DO; Christopher Halleman, DO
}

\section{PRACTICE POINTS}

- Argyria results from chronic exposure to products with a high silver content and may result in abnormalities of the skin and internal organs.

- Examination of the fingernails can provide important clues to underlying systemic conditions or external exposures.

To the Editor:

Argyria is a rare disease caused by chronic exposure to products with high silver content (eg, oral ingestion, inhalation, percutaneous absorption). With time, the blood levels of silver surpass the body's renal and hepatic excretory capacities that lead to silver granules being deposited in the skin and internal organs, including the liver, spleen, adrenal glands, and bone marrow. ${ }^{1}$ The cutaneous deposition results in a blue or blue-gray pigmentation of the skin, mucous membranes, and nails. Intervals of exposure that span from 8 months to 5 years prior to symptom onset have been described in the literature. ${ }^{2}$ The discoloration that results often is permanent, with no established way of effectively removing silver deposits from the tissue. ${ }^{3}$

A 22-year-old autistic man, who was completely dependent on his mother's care, presented to the emergency department with a primary concern of abdominal pain. The mother reported that he was indicating abdominal pain by motioning to his stomach for the last 5 days. The mother also reported he did not have a bowel movement during this time, and she noticed his hands were shaking. Prior to presentation, the mother had given him 2 enemas and had him on a 3-day strict liquid fast consisting of water, lemon juice, cayenne pepper, honey, and orange juice. Notably, the mother had a strong history of using naturopathic remedies for treatment of her son's ailments.

On admission, the patient was stable. There was a 2-point decrease in the patient's body mass index over the last month. Initial serum electrolytes were highly abnormal with a serum sodium level of $124 \mathrm{mEq} / \mathrm{L}$ (reference range, 135-145 mEq/L), blood urea nitrogen of $3 \mathrm{mg} / \mathrm{dL}$ (reference range, $7-20 \mathrm{mg} / \mathrm{dL}$ ), creatinine of $0.77 \mathrm{mg} / \mathrm{dL}$ (reference range, $0.74-1.35 \mathrm{mg} / \mathrm{dL}$ ), and lactic acid of $2.1 \mathrm{mEq} / \mathrm{L}$ (reference range, $0.5-1 \mathrm{mEq} / \mathrm{L}$ ). Serum osmolality was $272 \mathrm{mOsm} / \mathrm{kg}$ (reference range, 275-295 mOsm $/ \mathrm{kg}$ ). Urine osmolality was $114 \mathrm{mOsm} / \mathrm{kg}$ (reference range, 500-850 $\mathrm{mOsm} / \mathrm{kg}$ ) with a low-normal urine sodium level of $41 \mathrm{mmol} / 24 \mathrm{hr}$ (reference range, $40-220 \mathrm{mmol} / 24 \mathrm{hr}$ ). Abnormalities were felt to be secondary to malnutrition from the strict liquid diet (blood urea nitrogen and creatinine ratio of 3:1 suggestive of notable protein calorie malnutrition). The patient was given $1 \mathrm{~L}$ of normal saline in the emergency department, with further fluids held so as not to increase serum sodium level too rapidly. A regular diet was started.

Physical examination revealed dry mucosal membranes but otherwise was unremarkable. Active bowel sounds were noted, as well as a soft, nontender, and nondistended abdomen; however, when examining the patient's hands for reported shaking, a distinct abnormality of the nails was noticed. The patient had slate blue discoloration of the lunula, along with hyperpigmented violaceous discoloration of the proximal nail bed on all 10 fingernails (Figure 1). No abnormalities were seen on the toenails. The mother had a distinct bluish gray discoloration of the face as well as similar nail findings (Figure 2), strongly suggestive of colloidal silver use. An urgent serum silver level was ordered on the patient as well as a heavy metal panel. The mother was found applying numerous "natural remedies" to the patient's skin while in the hospital, including a liquid spray and lotion, both in unmarked bottles. At that time, the mother was informed that no external supplements should be applied to her son. The serum silver level was elevated substantially at $94.3 \mathrm{ng} / \mathrm{mL}$ (reference range, $<1.0 \mathrm{ng} / \mathrm{mL}$ ). When the mother was confronted, she initially denied use of silver but later admitted to notable silver content in the cream she was applying to her son's

Dr. Reader is from St. Joseph Mercy Health System, Ypsilanti, Michigan. Drs. Dillon and Halleman are from Largo Medical Center, Florida.

The authors report no conflict of interest.

Correspondence: Ashley M. Reader, DO (ashleyjag@gmail.com).

doi:10.12788/cutis.0282 


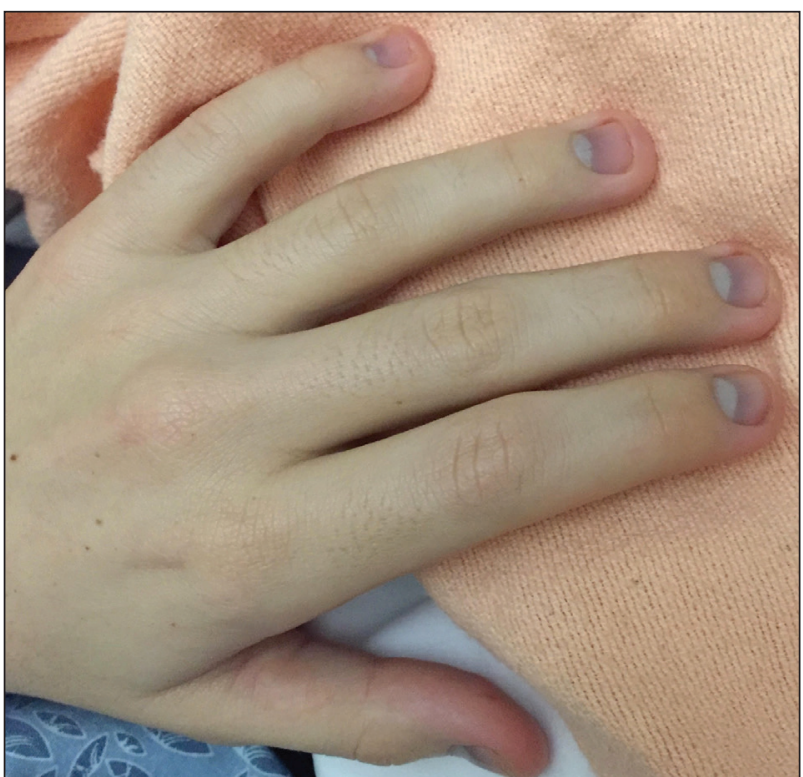

FIGURE 1. The fingernails on the patient's left hand exhibited slate blue discoloration of the lunula, along with hyperpigmented violaceous discoloration of the proximal nail bed. Similar findings were seen on the right hand.

skin. The mother reported that she read online that colloidal silver had been historically used to cure numerous ailments and she was ordering products from an online company. She was counseled on the dangers of both topical application and ingestion of silver, and all supplements were removed from the home.

Argyria is a rare condition caused by chronic exposure to silver and is characterized by a blue-gray pigmentation in the skin and appendages, mucous membranes, and internal organs. ${ }^{4}$ Clinically, argyria is classified as generalized or localized. Generalized argyria results from ingestion or inhalation of silver compounds, where granules deposit preferentially in sun-exposed areas of skin as well as internal organs, with the highest concentration in the liver, spleen, and adrenal glands; discoloration often is permanent. ${ }^{5}$ On the contrary, localized argyria results from direct external contact with silver and granules deposited in the hands, eyes, and mucosa. ${ }^{5}$ Although the exact mechanism of penetration from topical silver remains unknown, it is thought to enter via the eccrine sweat ducts, as histopathology reveals silver granules found in highest concentration surrounding sweat glands in the dermis. ${ }^{6}$

Initial differential diagnoses for altered nail pigmentation include drug-induced causes, systemic diseases, cyanosis, and exposure to metals. ${ }^{7}$ The most commonly indicated medications resulting in blue nail pigment changes include antimalarials, minocycline, zidovudine, and phenothiazine. Systemic diseases that may cause blue nail color change include Wilson disease, hemochromatosis, Addison disease, methemoglobinemia, and alkaptonuria. ${ }^{7}$ Metals include gold, mercury, arsenic,

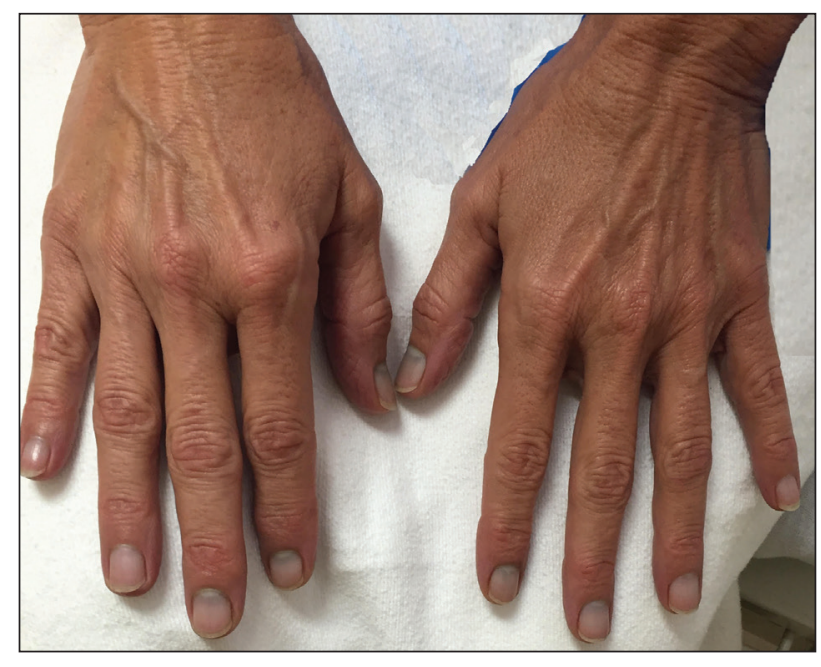

FIGURE 2. Similar, though less striking, nail changes were present on all 10 fingernails of the patient's mother.

bismuth, lead, and silver. ${ }^{4}$ After a thorough review of the patient's medications and lack of support for any underlying disease process, contact with metals, particularly silver, was ranked highly on our differential list. In support of this theory, the mother's bluish gray facial skin led to high clinical suspicion that she was ingesting colloidal silver and also was exposing her son to silver.

Treatment of argyria is challenging but first and foremost involves discontinuation of the source of chronic silver exposure. Unfortunately, the discoloration of generalized argyria often is permanent. Sunscreen can be used to help prevent any further darkening of pigment. The pigment in localized argyria has been reported to slowly fade with time, and there also have been reports of successful treatment using a low-fluence Q-switched 1064-nm Nd:YAG laser. ${ }^{8}$

\section{REFERENCES}

1. Molina-Hernandez AI, Diaz-Gonzalez JM, Saeb-Lima M, et al. Argyria after silver nitrate intake: case report and brief review of literature. Indian J Dermatol. 2015;60:520.

2. Lencastre A, Lobo M, João A. Argyria-case report. An Bras Dermatol. 2013;88:413-416.

3. Park S-W, Kim J-H, Shin H-T, et al. An effective modality for argyria treatment: Q-switched 1,064-nm Nd:YAG laser. Ann Dermatol. 2013;25:511-512.

4. Molina-Hernandez AI, Diaz-Gonzalez JM, Saeb-Lima M, et al. Argyria after silver nitrate intake: case report and brief review of literature. Indian J Dermatol. 2015;60:520.

5. Garcias-Ladaria J, Hernandez-Bel P, Torregrosa-Calatayud JL, et al. Localized cutaneous argyria: a report of 2 cases. Actas Dermosifiliogr. 2013;104:253-254.

6. Kapur N, Landon G, Yu RC. Localized argyria in an antique restorer. Br J Dermatol. 2001;144:191-192.

7. Kubba A, Kubba R, Batrani M, Pal T. Argyria an unrecognized cause of cutaneous pigmentation in Indian patients: a case series and review of the literature. Indian J Dermatol Venereol Leprol. 2013;79:805-811

8. Han TY, Chang HS, Lee HK, et al. Successful treatment of argyria using a low-fluence Q-switched 1064-nm Nd:YAG laser. Int J Dermatol. 2011;50:751-753. 